\title{
Cardiometabolic changes after continuous positive airway pressure for obstructive sleep apnoea: a randomised sham-controlled study
}

\author{
Camilla M Hoyos, ${ }^{1}$ Roo Killick, ${ }^{1}$ Brendon J Yee, ${ }^{1,2}$ Craig L Phillips, ${ }^{1,3}$ \\ Ronald R Grunstein, ${ }^{2,4}$ Peter Y Liu ${ }^{1,5}$
}

\begin{abstract}
- An additional material is published online only. To view this file please visit the journal online (http://dx.doi.org/10. 1136/thoraxjnl-2011-201420).

${ }^{1}$ Endocrine and Cardiometabolic Research Group, NHMRC Centre for Integrated Research and Understanding of Sleep (CIRUS). Woolcock Institute of Medical Research, University of Sydney, Glebe, Australia

${ }^{2}$ Sleep and Circadian Research Group, NHMRC Centre for Integrated Research and Understanding of Sleep (CIRUS) Woolcock Institute of Medical Research, University of Sydney, Glebe, Australia

${ }^{3}$ Royal North Shore Hospital, Sydney, Australia

${ }^{4}$ Royal Prince Alfred Hospital,

Sydney, Australia

${ }^{5}$ Division of Endocrinology, Department of Medicine, Harbor-UCLA Medical Center and Los Angeles Biomedical Research Institute, Torrance, California, USA
\end{abstract}

\section{Correspondence to}

Dr Peter Y Liu, Endocrine and Cardiometabolic Research

Group, Woolcock Institute of Medical Research, University of Sydney, Glebe, NSW 2037. Australia;

pliu@mail.usyd.edu.au

Received 28 November 2011 Accepted 7 April 2012

Published Online First 5 May 2012

\section{ABSTRACT}

Rationale and objectives Impaired insulin sensitivity (ISx), increased visceral abdominal fat (VAF) and liver fat are all central components of the metabolic syndrome and characteristics of men with obstructive sleep apnoea (OSA). The reversibility of these observed changes with continuous positive airway pressure (CPAP) treatment in men with OSA has not been systematically studied in a randomised sham-controlled fashion.

Methods 65 men without diabetes who were CPAP naïve and had moderate to severe OSA (age $=49 \pm 12$ years, apnoea hypopnoea index $(A H I)=$ $39.9 \pm 17.7$ events $/ \mathrm{h}$, body mass index $=31.3 \pm 5.2 \mathrm{~kg} /$ $\left.\mathrm{m}^{2}\right)$ were randomised to receive either real $(n=34)$ or sham ( $n=31)$ CPAP for 12 weeks. At 12 weeks, all subjects received real CPAP for an additional 12 weeks. Measurements and main results Main outcomes were the change at week 12 from baseline in VAF, ISx and liver fat. Other metabolic outcomes were changes in the disposition index, total fat, and blood leptin and adiponectin concentrations. The AHI was lower on CPAP compared with sham by 33 events/h $(95 \% \mathrm{Cl}-43.9$ to -22.2 , $\mathrm{p}<0.0001)$ after 12 weeks. There were no between-group differences at 12 weeks in VAF $\left(-13.0 \mathrm{~cm}^{3},-42.4\right.$ to 16.2, $\mathrm{p}=0.37)$, ISx $\left(-0.13\left(\mathrm{~min}^{-1}\right)(\mu \mathrm{U} / \mathrm{ml})\right)^{-1},-0.40$ to $0.14, p=0.33)$, liver fat $\left(-0.5 \mathrm{~cm}^{3},-3.8\right.$ to $\left.2.7, p=0.74\right)$ or any other cardiometabolic parameter. At 24 weeks, ISx $\left(3.2 \times 10^{4}\left(\mathrm{~min}^{-1}\right)(\mu \mathrm{U} / \mathrm{ml})\right)^{-1}, 0.9 \times 10^{4}$ to $6.0 \times 10^{4}$, $\mathrm{p}=0.009)$, but not VAF $\left(-1.4 \mathrm{~cm}^{3},-19.2\right.$ to 16.4 , $p=0.87)$ or liver fat $(-0.2$ Hounsfield units, -2.4 to $2.0, p=0.83$ ) were improved compared with baseline in the whole study group.

Conclusion Reducing visceral adiposity in men with OSA cannot be achieved with CPAP alone and is likely to require weight-loss interventions. Longer-term effects of CPAP on other cardiometabolic markers such as ISx require further investigation to fully examine time dependencies.

Trial Registration Number ACTRN12608000301369.

\section{INTRODUCTION}

Obstructive sleep apnoea (OSA) is a common disorder that affects up to $25 \%$ of adult men. ${ }^{1}$ Untreated severe OSA increases the risk of all-cause and cardiovascular mortality. ${ }^{2-4}$ It is generally believed that these increased risks are partly due to obesity, increased visceral abdominal fat (VAF), insulin resistance (IR) and increased liver fat. ${ }^{5}$ This is because VAF, liver fat and IR (central components
Key messages

What is the key question?

- Does continuous positive airway pressure therapy, as practically applied in the community, improve central parameters of cardiometabolic risk in men with obstructive sleep apnoea?

What is the bottom line?

- We found that CPAP may improve insulin sensitivity but does not decrease visceral adiposity or liver fat by 24 weeks.

Why read on?

- Our results are of relevance to sleep medicine physicians who now cannot rely solely on CPAP therapy to improve cardiometabolic health. Weight loss interventions are likely to be needed to reduce visceral adiposity in men with obstructive sleep apnoea.

of the metabolic syndrome) are all independently associated with increased risk for cardiovascular disease and mortality in adults not selected for OSA. $^{6-8}$ Furthermore, long-term CPAP treatment decreases mortality risk, ${ }^{9}$ but whether this is due to CPAP-induced improvements in IR or body fat distribution, including decreases in VAF and liver fat, has not been systematically studied. Indeed, the reversibility of IR, VAF and liver fat in OSA is still not known because few randomised controlled CPAP intervention studies have been performed.

Randomised studies investigating the effect of CPAP on VAF or liver fat are not yet available. However, we $e^{10}$ and others ${ }^{11}$ previously reported significant reductions in VAF volume after 3 and 6 months of CPAP treatment respectively, although other studies did not detect such changes. ${ }^{12-14} \mathrm{~A}$ single long-term (2-3 years) uncontrolled study showed that CPAP reduced liver fat but only in those who were CPAP compliant $(n=6) .{ }^{15}$ Randomised controlled trials are required to fully address the question of whether CPAP alters VAF and liver fat.

Three randomised sham-controlled trials have examined the effect of CPAP therapy on IR, but the results are inconclusive. Only one of the three studies reported a statistically significant improvement in IR, but the controlled portion of this study was of 1 week duration, so that the durability of 
these findings is not known. ${ }^{14}$ The other two studies did not show any change in IR with CPAP. However, one was performed specifically in diabetic men in whom the extent of reversibility may be limited ${ }^{16}$ and the other did not assess insulin resistance by a sensitive method. ${ }^{17}$ Additionally none of these three studies examined other important measures of glucose metabolism such as the disposition index (DI), a measure which combines impaired $\beta$-cell activity with $\operatorname{IR}^{18}$ and is useful in predicting future diabetes. ${ }^{19}$ Furthermore, the important inter-relationships among VAF, liver fat and IR have not been investigated because no randomised controlled trial has measured all of these important biomarkers concurrently.

We therefore undertook a randomised sham-controlled trial to determine the effects of 12 weeks of CPAP treatment on VAF and liver fat (CT scans), insulin sensitivity (ISx) and DI (from minimal model analysis) in men with OSA without known diabetes. We also measured total body fat and muscle mass (whole body dual-energy X-ray absorptiometry (DEXA) scans), leptin and adiponectin concentrations. All subjects received an additional 12 weeks of open-label CPAP treatment after which all outcomes were remeasured.

\section{METHODS}

\section{Setting and participants}

Participants were recruited from tertiary referral sleep clinics at Royal Prince Alfred Hospital and the Woolcock Institute of Medical Research, Sydney, Australia between July 2008 and December 2010. Eligible participants were adult men with moderate-severe OSA, defined as aged $\geq 18$ years, an apnoea hypopnoea index (AHI) $\geq 20$ events/ $h$ and an oxygen desaturation index $3 \% \geq 15$ events/h measured by in-laboratory polysomnography (PSG). Participants were excluded if they had diagnosed type II diabetes mellitus; previously used CPAP treatment; minimum oxygen saturation $<65 \%$ or an AHI $>80$ events/h or required immediate CPAP treatment due to excessive sleepiness in relation to the subject's occupation; uncontrolled concurrent medical, drug abuse or psychiatric illness; contraindication to CPAP therapy; irregular sleep patterns such as shift workers; or participation in another clinical trial in the previous 30 days.

\section{Design overview}

This was a randomised, double-blind, sham-controlled, parallelgroup study. Following baseline data collection, eligible participants were randomised to receive either real or sham CPAP for 12 weeks. Clinical assessments were performed before, midway at 6 weeks, and after treatment. At the end of the 12-week blinded period all participants were provided with open-label real CPAP for an additional 12 weeks. Assessments were recollected at 24 weeks.

The study complied with Good Clinical Practice guidelines, applicable regulatory requirements and the Declaration of Helsinki. All participants provided written informed consent to participate in the study, which was approved by the Sydney South West Area Health Service Human Research and Ethics Committee (RPAH Zone). The study is registered with the Australia New Zealand Clinical Trials Network, http://www. anzctr.org.au, number ACTRN12608000301369.

\section{Randomisation, allocation concealment and blinding}

A computer programme produced randomised permuted blocks with a block size of four. Participants were assigned to real or sham CPAP in a 1:1 ratio. At baseline each participant was assigned a unique number in sequential, ascending, chronological order which corresponded to the treatment allocation. Machine preparation was performed by a person separate to the study investigators and not involved in participant assessments. The study investigators were blinded to treatment allocation for the duration of the study. During the open-label period both the participants and study investigators remained blinded to the initial treatment allocation.

\section{Outcomes}

The predefined primary outcome was the change in VAF from baseline to week 12 . The main predefined secondary outcome was the change in ISx from baseline to week 12. Other secondary outcomes were the changes from baseline to week 12 in liver fat, body composition (weight, body mass index (BMI), waist circumference, abdominal subcutaneous fat, total fat and lean mass) and metabolic markers (DI, homeostasis model assessment (HOMA), quantitative insulin sensitive check index (QUICKI), fasting glucose and insulin, leptin and adiponectin). We also assessed the change from baseline to week 24 in all measures.

\section{CPAP machines and titration}

The real and sham CPAP machines (Remstar Auto, Philips Respironics, Murrysville, Pennsylvania, USA) were identical in appearance to each other and have been used previously at our centre $^{20}$ and by others. ${ }^{21}$ The sham device delivered airflow with minimal pressure $(0.5 \mathrm{~cm} \mathrm{H} \mathrm{O})$. Prior to randomisation every subject was fitted with a mask and received a standard CPAP education programme operating in our clinic. Compliance data were recorded by an internal clock within all real and sham CPAP machines and were downloaded after the home titration and at each visit. Further details are available in the online supplement.

\section{PSG and sleepiness}

Sleep and breathing were assessed by attended overnight, inlaboratory PSG (Sandman Elite V.9.2, Tyco Healthcare, Denver, Colorado, USA). A standard diagnostic PSG was performed at week 0. At weeks 12 and 24, standard PSGs were recorded in all participants irrespective of whether the participant was using real or sham CPAP. Subjective sleepiness was measured by the Epworth Sleepiness Scale (ESS) ${ }^{22}$ at all visits. Further details are available in the online supplement.

\section{VAF and body composition}

VAF and subcutaneous fat were measured by CT (16-slice light speed extra; GE Healthcare, Madison, Wisconsin, USA). The volumes of VAF and subcutaneous fat were calculated using a validated software tool (Hippofat; CNR Institute of Clinical Physiology, Pisa, Italy) ${ }^{23}$ from two $10 \mathrm{~mm}$ contiguous CT images of the lower abdomen, at the level of the umbilicus, between the L4 and L5 vertebra. Liver fat, estimated by liver and spleen Hounsfield unit (HU) attenuations (E-FILM, Merge Healthcare, Milwaukee, Wisconsin, USA) was quantified from unenhanced CT slices by a previously published method. ${ }^{24}$ Total fat and muscle mass were measured by total body DEXA (Lunar Prodigy; GE Healthcare). All scans were performed at 0, 12 and 24 weeks. Anthropometric measurements were also recorded using standardised techniques at each visit by a single observer. ${ }^{25}$

\section{Measures of metabolic dysfunction}

ISx and the DI were calculated by minimal model. ${ }^{26}$ Venous blood samples were collected via a cannula following an overnight fast and a standard 3-day carbohydrate-loaded diet at 0, 12 and 24 weeks. A fasting blood sample was first collected and 
then at 10, 20, 30, 60, 75, 90 and $120 \mathrm{~min}$ after a $75 \mathrm{~g} / 300 \mathrm{ml}$ oral glucose load following an established protocol. ${ }^{26}$ Additionally fasting samples were collected at $0,6,12,24$ weeks and the $\mathrm{HOMA}^{27}$ and QUICKI ${ }^{28}$ were calculated. Glucose, insulin, C peptide, leptin and adiponectin concentrations were all measured with commercially available assays. All samples were stored at $-80^{\circ} \mathrm{C}$ for subsequent batched analysis and all samples from an individual patient were run within a single assay.

\section{Statistical analysis}

The study was powered to detect a standardised mean change in VAF of $0.78 \pm 1$ using our previously published data. ${ }^{10}$ The total sample size (assuming 1:1 randomisation) required to detect this effect with $80 \%$ power at a two-tailed significance level of $5 \%$ was 52 men treated for 12 weeks.

The outcome variables were the calculated differences from baseline at 6, 12 and 24 weeks. Linear regression was used to determine between-treatment group differences of these calculated differences from baseline during the blinded period ( 6 and 12 weeks). Mixed model analysis was used for outcomes with repeated measurements. Further analyses explored the influence of treatment compliance, baseline severity (AHI), obesity (BMI and waist circumference) and sleepiness (ESS). These potential confounders were included as linear covariates. Additionally covariates with predefined cut points were included in separate mixed models as a dichotomised factor (compliance $4 \mathrm{~h} /$ night; AHI 30 events/h; BMI $30 \mathrm{~kg} / \mathrm{m}^{2} ;$ ESS 10). The statistical significance of the interaction terms of treatment and each dichotomised variable were examined. These analyses were performed to assess whether the effect of CPAP was influenced by compliance, baseline AHI, obesity or sleepiness. Pooled mean changes and the SE of the mean from baseline to week 24 were determined regardless of initial treatment allocation. Unpooled mean changes are also shown and analysed analogously.

Analyses were performed using SAS V.9.2 (SAS Institute). Data were considered significantly different at $p<0.05$ (two sided) and are presented as mean differences (95\% CI), mean (SD), or median (IOR) as indicated.

\section{RESULTS}

The flow of participants through the study is shown in figure 1. Sixty-nine men were enrolled of whom 65 were randomised to receive either real $(n=34)$ or sham $(n=31)$ CPAP treatment. Four participants could not complete home titration after multiple attempts and two other participants withdrew for personal reasons prior to starting real or sham CPAP (figure 1). Primary outcome data were available for 52 men at week 12 and 46 men at week 24. Baseline characteristics were comparable in the two treatment groups (table 1). The withdrawn subjects were comparable to those who completed, except for a significantly lower CPAP adherence rate (data not shown). None of the participants had physician-diagnosed diabetes at baseline, 92\% were overweight, $70 \%$ had abdominal obesity and $55 \%$ were medically obese. Two participants without diagnosed diabetes during screening were randomised, and subsequently discovered to have diabetes after analysis of the oral glucose tolerance test at the end of the study. ${ }^{29}$ The analyses were performed with and without these participants and there was no difference in findings. All findings presented here are of all participants with available data.

During the blinded period, real CPAP use was $3.6 \mathrm{~h}$ per night and sham CPAP use was $2.8 \mathrm{~h}$ per night $(\mathrm{p}=0.07)$. During the open-label period, CPAP use was $4 \mathrm{~h}$ per night for both groups $(p=0.72)$. CPAP use did not change during the open-label period in those initially randomised to CPAP therapy $(p=0.16)$ but increased by $1.1 \mathrm{~h}$ in those initially randomised to sham therapy $(p=0.04)$. All participants with available data, regardless of CPAP use, were included in the analysis.

The change in VAF or any other measurements of body composition at week 12 were not significantly different between groups (figure 2). At week 12 there was a within-group increase in lean muscle mass in the CPAP group $(p=0.001)$ compared
Figure 1 Study flow. CPAP, continuous positive airway pressure.

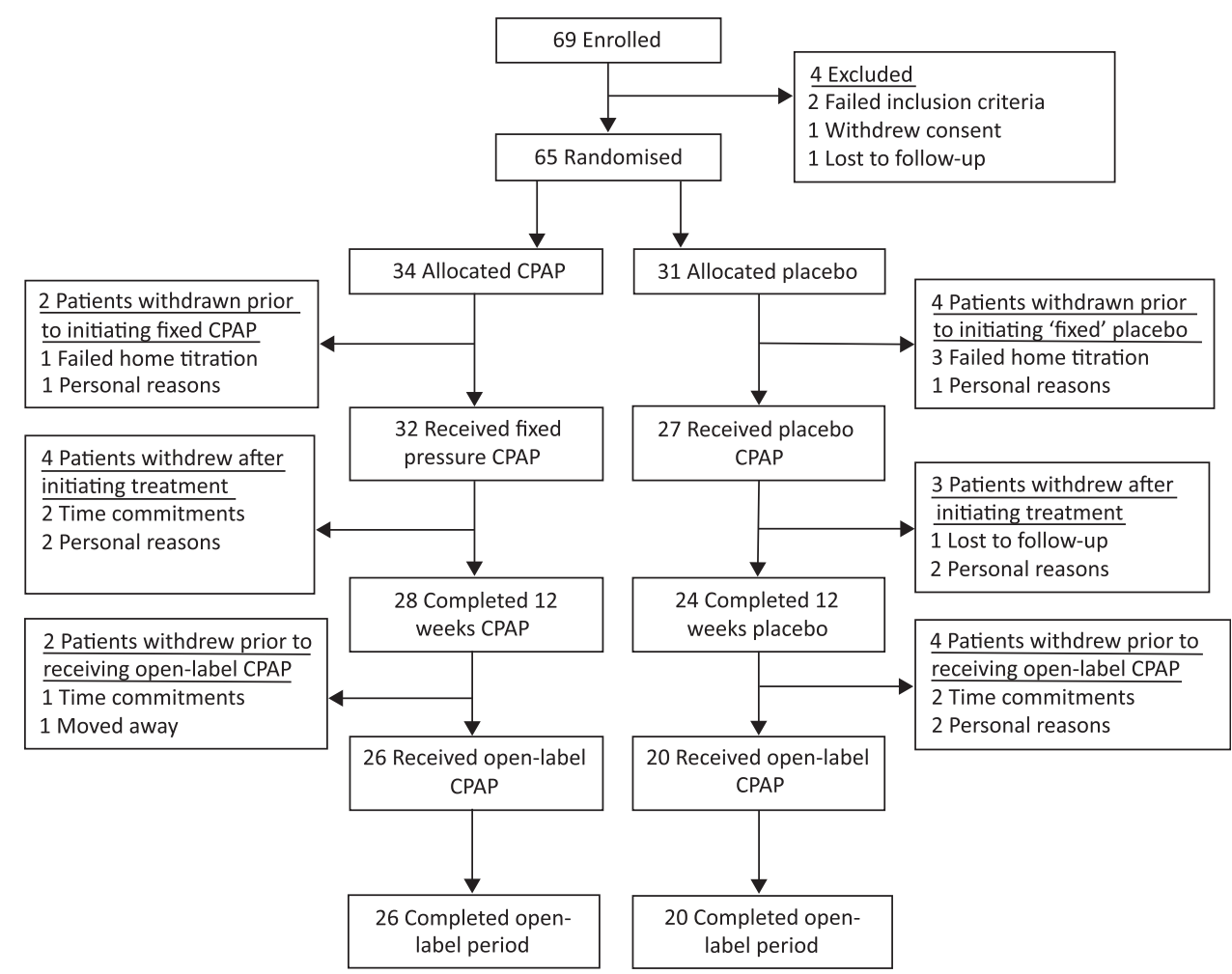


Table 1 Baseline participant characteristics

\begin{tabular}{|c|c|c|c|}
\hline & Real CPAP $(n=34)$ & Sham CPAP $(n=31)$ & p Value \\
\hline Age (years) & $51.0(12.3)$ & $46.4(10.4)$ & 0.11 \\
\hline \multicolumn{4}{|l|}{ Sleep and breathing } \\
\hline TST (min) & 362 (308 to 400$)$ & $378(329$ to 406$)$ & $0.19^{*}$ \\
\hline Sleep efficiency (\%) & 81.2 (67.4 to 88.7 ) & 85.3 (76.9 to 92.3 ) & $0.09^{*}$ \\
\hline REM (\% TST) & $14.0(5.0)$ & $17.0(6.0)$ & 0.08 \\
\hline Non-REM (\% TST) & $86.0(5.0)$ & $83.0(6.0)$ & 0.09 \\
\hline SWS (\% TST) & $17.7(11.3)$ & $21.0(8.6)$ & 0.10 \\
\hline AHI (events/h) & $38.5(14.7)$ & $41.5(20.7)$ & 0.79 \\
\hline ODI3 (events/h) & $32.0(16.8)$ & $34.9(17.6)$ & 0.74 \\
\hline Minimum saturation $(\%)$ & 82 (79 to 85$)$ & 79 (74 to 84$)$ & $0.12^{*}$ \\
\hline $\mathrm{SpO}_{2} \mathrm{~T} 90(\% \mathrm{TST})$ & $3.1(1.0$ to 5.6$)$ & $3.6(1.2$ to 8.2$)$ & $0.54^{*}$ \\
\hline \multicolumn{4}{|l|}{ Sleepiness } \\
\hline Subjective sleepiness (ESS, scale 0-24) & $10.0(4.0)$ & $10.2(4.8)$ & 0.82 \\
\hline \multicolumn{4}{|l|}{ Anthropometry } \\
\hline Weight (kg) & $97.4(20.7)$ & $94.3(17.9)$ & 0.53 \\
\hline BMI $\left(\mathrm{kg} / \mathrm{m}^{2}\right)$ & $31.6(5.3)$ & $31.0(5.1)$ & 0.63 \\
\hline Waist circumference $(\mathrm{cm})$ & $109.7(13.3)$ & $106.3(13.0)$ & 0.31 \\
\hline \multicolumn{4}{|l|}{ Body composition } \\
\hline Visceral abdominal fat $\left(\mathrm{cm}^{3}\right)$ & $307.4(118.2)$ & $299.2(126.0)$ & 0.72 \\
\hline Subcutaneous abdominal fat $\left(\mathrm{cm}^{3}\right)$ & $669.2(239.7)$ & $571.1(225.4)$ & 0.23 \\
\hline Liver fat (HU) & $52.6(13.3)$ & $52.9(9.8)$ & 0.91 \\
\hline $\mathrm{L} / \mathrm{S}$ ratio $(\mathrm{HU})$ & $1.1(0.2)$ & $1.0(0.2)$ & 0.19 \\
\hline Total fat $(\mathrm{kg})$ & $33.4(12.6)$ & $29.9(10.8)$ & 0.25 \\
\hline Total lean muscle $(\mathrm{kg})$ & $59.6(9.1)$ & $59.7(8.6)$ & 0.98 \\
\hline \multicolumn{4}{|l|}{ Metabolic markers } \\
\hline $\mathrm{ISx}\left(\min ^{-1}\right)(\mu \mathrm{U} / \mathrm{ml})^{-1}$ & $7.1 \times 10^{4}\left(5.8 \times 10^{4}\right)$ & $5.5 \times 10^{4}\left(4.8 \times 10^{4}\right)$ & 0.44 \\
\hline DI (unit) & $0.07(0.09)$ & $0.05(0.07)$ & 0.26 \\
\hline HOMA (unit) & $2.9(2.5)$ & $2.9(1.8)$ & 0.70 \\
\hline QUICKI (unit) & $0.3(0.02)$ & $0.4(0.05)$ & 0.36 \\
\hline Fasting glucose (mmol/litre) & $5.3(0.7)$ & $5.2(0.5)$ & 0.56 \\
\hline Fasting insulin $(\mu \mathrm{lU} / \mathrm{ml})$ & $11.8(7.8)$ & $12.5(7.7)$ & 0.81 \\
\hline Adiponectin (ng/ml) & $7.3(3.4)$ & $7.0(3.6)$ & 0.59 \\
\hline Leptin (ng/ml) & $15.9(9.8)$ & $14.5(10.6)$ & 0.58 \\
\hline \multicolumn{4}{|l|}{ Medical history } \\
\hline Hypertension, $\mathrm{n}(\%)$ & $11(32)$ & $11(35)$ & 0.80 \\
\hline Using antihypertensive, $\mathrm{n}(\%)$ & $11(32)$ & $11(35)$ & 0.80 \\
\hline Hypercholesterolaemia, n (\%) & $12(35)$ & $12(39)$ & 0.80 \\
\hline Using statins, $\mathrm{n}(\%)$ & $11(32)$ & $7(23)$ & 0.42 \\
\hline Pre-existing CVD, n (\%) & $5(15)$ & $3(10)$ & 0.71 \\
\hline Glucose intolerance, $† \mathrm{n}(\%)$ & $10(29)$ & $7(23)$ & 0.58 \\
\hline
\end{tabular}

Values are mean (SD), median (IQR) or number (\%) as appropriate. Between-group differences were assessed by $t$ test after normalisation as required, or by rank-sum test as indicated with *.

tDetermined by oral glucose tolerance test.

AHI, apnoea-hypopnoea index; BMI, body mass index; CPAP, continuous positive airway pressure; CVD, cardiovascular disease; DI, insulin disposition index measured by minimal model analysis; ESS, Epworth Sleepiness Scale; HOMA, homeostasis model assessment of insulin resistance; HU, Hounsfield unit; ISx, insulin sensitivity measured by minimal model analysis; L/S, liver/spleen; ODI3, oxygen desaturation index $3 \%$ pressure; QUICKI, quantitative insulin sensitivity check index; REM, rapid eye movement; $\mathrm{SpO}_{2} \mathrm{~T} 90$, time that the arterial oxygen saturation was $<90 \%$; TST, total sleep time; SWS, slow wave sleep.

with a non-significant change after sham treatment $(p=0.20)$. At week 24 , lean muscle had increased in all participants but there were no changes in any other measures of body composition (figure 2 and table 3). Furthermore, the actual decrease in VAF at week 24 was $1.4 \mathrm{~cm}^{3}$ which corresponds to a standardised mean change in VAF of $0.08 \pm 1$ (table 3 ).

There were no significant changes in ISx, DI, HOMA, QUICKI, fasting glucose, insulin, adiponectin or leptin over the first 12 weeks within or between treatment groups (figures 3 and 4). At week 24, the entire pooled group improved in ISx, as measured by minimal model analysis (figure $3 \mathrm{~A}$ ). These improvements were due to a significant increase in those initially randomised to real CPAP $(\mathrm{p}=0.02)$, rather than sham $(p=0.20)$ (table 3$)$, and there was no between-group difference $(p=0.32)$. There were no other significant changes in metabolic parameters (figures 3 and 4).
At week 12, real CPAP treatment significantly improved OSA parameters compared with sham CPAP (figure 5). The average nightly AHI as measured by the CPAP device, over the entire 12-week period, was 3.1 events/h in the real CPAP group (not available for sham group). The change in total sleep time was not different between groups, but rapid eye movement (REM) and non-REM sleep significantly increased and decreased respectively with real CPAP compared with sham (table 2). There were no between-group differences in the changes in subjective sleepiness (table 2). At week 24, OSA parameters had improved from baseline in all participants regardless of initial treatment allocation (figure 5 and table 3 ).

We next assessed whether CPAP compliance, baseline age, AHI, BMI, waist circumference or sleepiness influenced treatment outcomes (namely, change in VAF, ISx and liver fat). We dichotomised age by the median, compliance by $4 \mathrm{~h} /$ night, AHI 

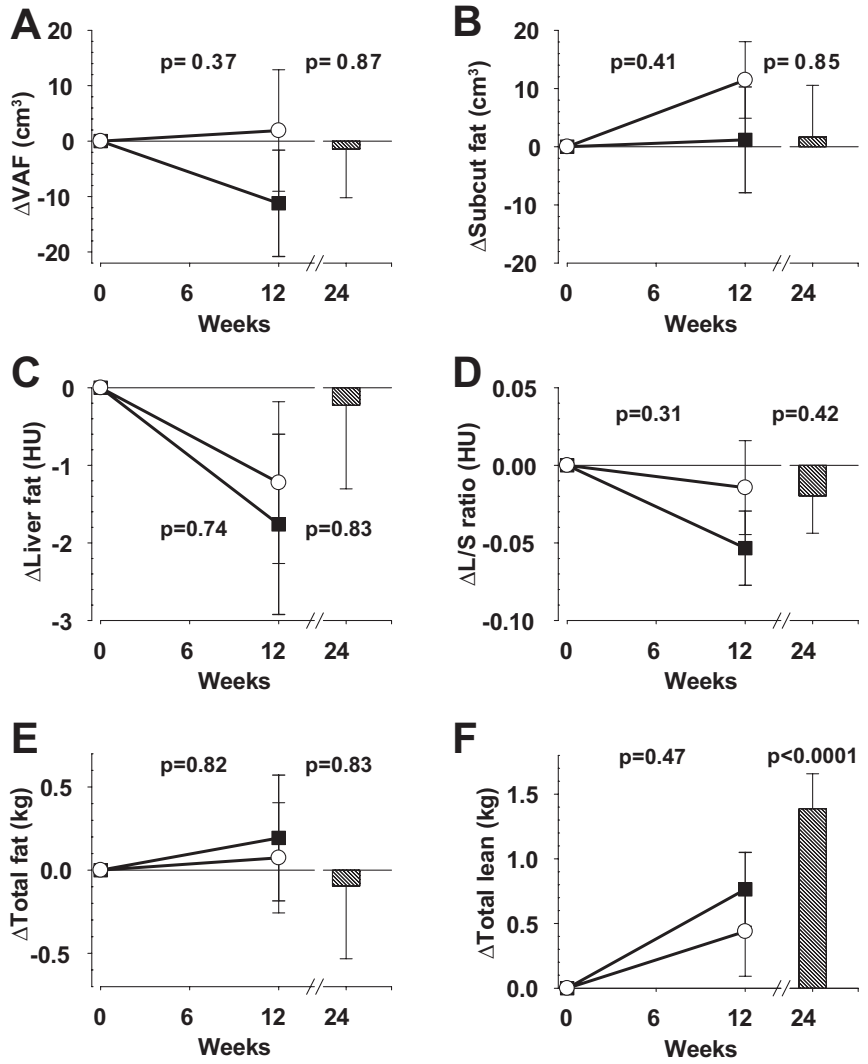

$\mathbf{F}$

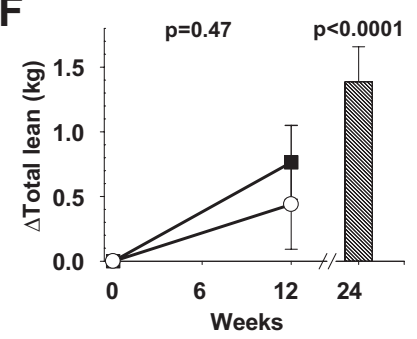

A
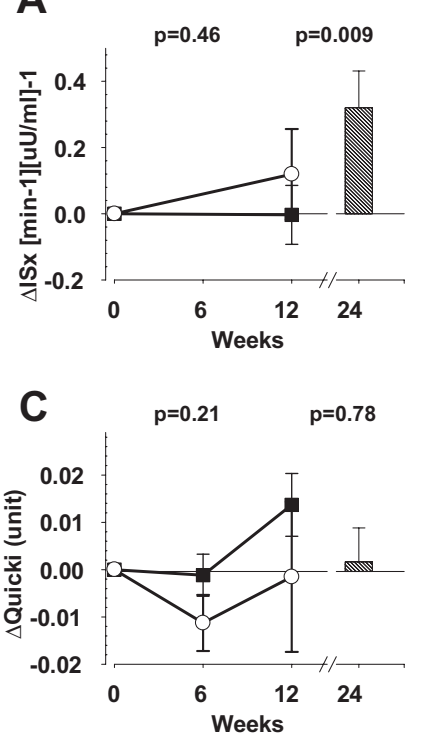

B

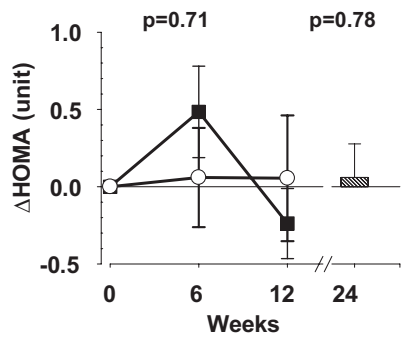

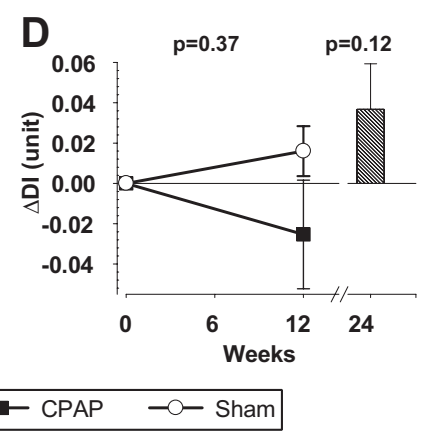

Figure 3 Plot of mean and SE of the mean for $(A)$ insulin sensitivity as measured by minimal model analysis (ISx), (B) insulin resistance as measured by the homeostatic model (HOMA), (C) insulin sensitivity measured by the quantitative insulin sensitive check index (QUICKI) and (D) disposition index. The left-hand plot (line graph) shows the mean change at week 12 (and week 6 in plots B and C) from baseline for the real continuous positive airway pressure (CPAP) (filled circles) and sham CPAP (open circles) groups. The $\mathrm{p}$ value above this graph denotes the between-group difference as determined by linear regression (mixed model analysis for plots $B$ and C). The right-hand plot (vertical bar graph) is the pooled mean change at week 24 from baseline in all participants regardless of initial treatment allocation. The $p$ value above this graph denotes the significance of the change as determined by Student's t test.

tion (week 12: $r^{2}=0.33, p=0.0008$; week 24: $r^{2}=0.55, p=0.0001$ ). There was no such correlation between BMI and adiponectin.

\section{DISCUSSION}

This is, to our knowledge, the first randomised sham-controlled trial of the effect of CPAP treatment for OSA on VAF and liver fat, and the largest and longest trial of CPAP on ISx to date. We demonstrated that 12 weeks of CPAP did not improve VAF, liver fat or ISx compared with sham CPAP. Small long-term improvements in ISx, but not VAF or liver fat, were observed but only in those who received a total of 6 months of CPAP treatment, albeit in a non-randomised phase. Furthermore, both the actual and standardised mean changes from baseline in VAF at 24 weeks were small. These data suggest that reducing visceral adiposity cannot be achieved with CPAP alone and that other interventions to reduce VAF will be required. This is important because reducing abdominal adiposity, rather than overall adiposity, is now recognised as the more metabolically relevant target. At present, lifestyle modification programmes to enforce total weight loss would seem to be the most likely strategy to ensure regional changes in adiposity and such programmes have been successfully applied in those with OSA. ${ }^{30-32}$ Pharmaceutical methods could still be used in conjunction with lifestyle modification, but available options are becoming increasingly limited and the safety of any new drug would need to be firmly established in the OSA population. ${ }^{33}$

Our finding that CPAP does not alter VAF after at least 3 months of CPAP treatment is consistent with uncontrolled

At weeks 12 and 24 there was a significant correlation between change in BMI with the change in leptin concentrations in the whole group regardless of initial treatment alloca- 
A
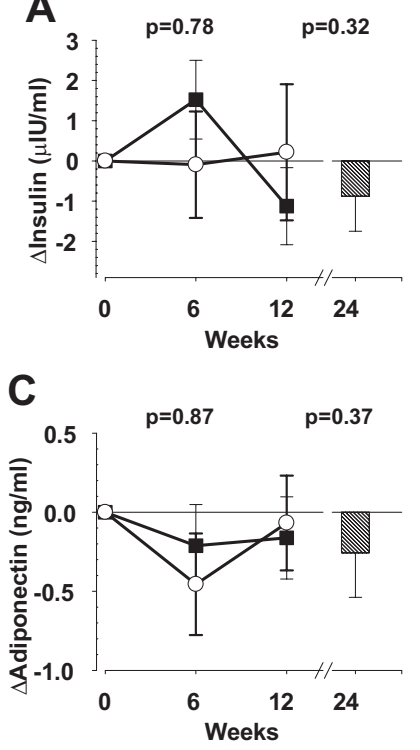

B

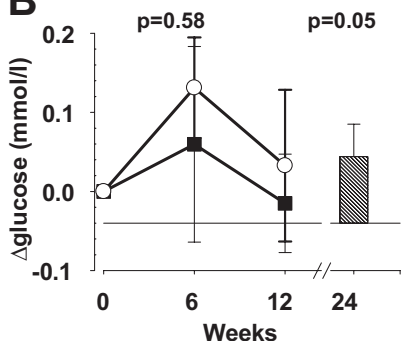

D

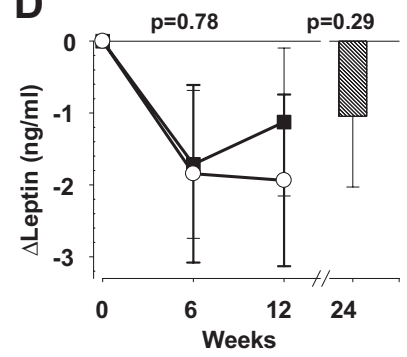

A

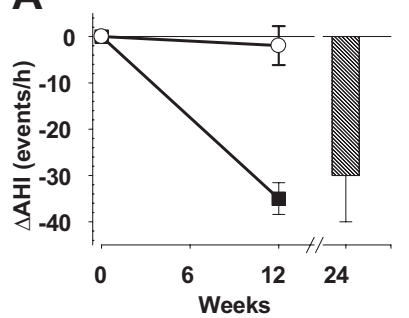

C

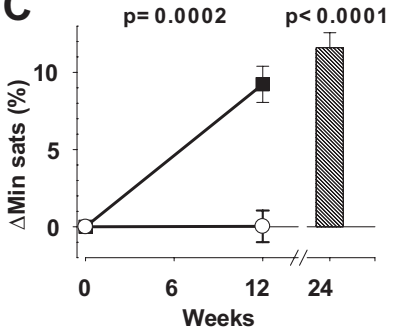

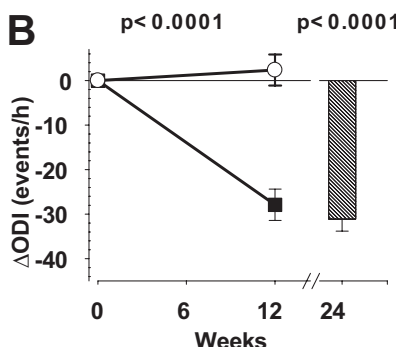

D $p=0.02 p=0.001$

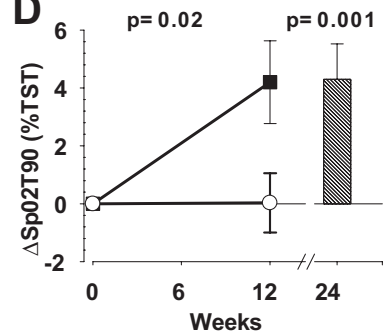

mimmm Combined $\rightarrow$ CPAP $-\circ-$ Sham

Figure 5 Plot of mean and SE of the mean for (A) apnoea hypopnoea index (AHI), (B) oxygen desaturation index (ODI), (C) minimum saturation level (min sats) and (D) the per cent of total sleep time that the arterial oxygen saturation was $<90 \%\left(\mathrm{SpO}_{2} \mathrm{~T} 90\right)$. The left-hand plot (line graph) shows the mean change at week 12 from baseline for the real continuous positive airway pressure (CPAP) (filled circles) and sham CPAP (open circles) groups. The $p$ value above this graph denotes the between-group difference as determined by linear regression. The righthand plot (vertical bar graph) is the pooled mean change at week 24 from baseline in all participants regardless of initial treatment allocation. The $p$ value above this graph denotes the significance of the change as determined by Student's t test.

ment duration as the two uncontrolled and non-randomised studies which previously reported that CPAP decreases VAF. ${ }^{10} 11$ Furthermore, the recently published randomised shamcontrolled CPAP study showed that CPAP reduced VAF, total body weight and metabolic syndrome. ${ }^{34} \mathrm{~A}$ reduction in total

studies, including the longest ( 8 months) and largest $(n=113)$ to date. ${ }^{12-14}$ In contrast, a recent 3 -month randomised shamcontrolled study, ${ }^{34}$ as well as a 3 -month ${ }^{10}$ and 6 -month ${ }^{11}$ uncontrolled study have shown that CPAP reduces VAF. Our study is more than twice as large and of equal or longer treat-

Table 2 Changes from baseline in real CPAP and sham CPAP groups and the between-group differences

\begin{tabular}{|c|c|c|c|c|}
\hline & \multicolumn{2}{|l|}{ Mean change } & \multirow{2}{*}{$\begin{array}{l}\text { Between-group differences } \\
\text { (real - sham) } \\
\text { Mean difference }(95 \% \mathrm{CI})\end{array}$} & \multirow[b]{2}{*}{ p Value } \\
\hline & Real CPAP $(n=26)$ & Sham CPAP $(n=24)$ & & \\
\hline \multicolumn{5}{|l|}{ Sleep architecture and sleepiness } \\
\hline TST (min) & 25.2 & 26.9 & $-1.7(-43.2$ to 39.9$)$ & 0.94 \\
\hline Sleep efficiency (\% TST) & 4.8 & 2.8 & $2.0(-5.9$ to 9.8$)$ & 0.62 \\
\hline REM (\% TST) & 0.04 & -0.02 & $0.06(0.01$ to 0.1$)$ & 0.02 \\
\hline Non-REM (\% TST) & -0.04 & 0.02 & $-0.06(-0.1$ to -0.01$)$ & 0.02 \\
\hline SWS (\% TST) & 11.6 & 4.5 & $7.1(-0.04$ to 14.3$)$ & 0.05 \\
\hline Subjective sleepiness (ESS, scale 0-18) & -1.8 & -1.7 & $-0.1(-1.6$ to 1.9$)$ & 0.87 \\
\hline Objective sleepiness (OSLER, min) & 4.5 & 3.2 & $1.3(-2.6$ to 5.3$)$ & 0.19 \\
\hline \multicolumn{5}{|l|}{ Anthropometry } \\
\hline BMI $\left(\mathrm{kg} / \mathrm{h}^{2}\right)$ & 0.14 & 0.08 & $0.06(-0.3$ to 0.4$)$ & 0.71 \\
\hline Weight (kg) & 0.5 & 0.3 & $0.3(-0.7$ to 1.2$)$ & 0.57 \\
\hline Waist circumference $(\mathrm{cm})$ & -0.5 & -0.7 & $0.2(-1.2$ to 1.6$)$ & 0.79 \\
\hline \multicolumn{5}{|l|}{ Clinic blood pressure } \\
\hline $\mathrm{SBP}(\mathrm{mm} \mathrm{Hg})$ & -4.75 & -0.50 & $-4.25(-9.60$ to 1.10$)$ & 0.12 \\
\hline $\mathrm{DBP}(\mathrm{mm} \mathrm{Hg})$ & 0.41 & -0.04 & $0.45(-3.38$ to 4.28$)$ & 0.82 \\
\hline HR (beats/min) & -1.48 & 1.38 & $-2.86(-7.17$ to 1.45$)$ & 0.19 \\
\hline MAP (mm Hg) & -1.32 & -0.30 & $-1.01(-4.49$ to 2.47$)$ & 0.56 \\
\hline
\end{tabular}

Bold values are statistically significant $(p<0.05)$.

Data are mean differences from baseline, between-group differences (and $95 \%$ Cls) and unadjusted $p$ values. Between-group differences were determined by linear regression. Mixed model analysis was used for outcomes with repeated measures.

BMI, body mass index; CPAP, continuous positive airway pressure; DBP, diastolic blood pressure; ESS, Epworth Sleepiness Scale; HR, heart rate; MAP, mean arterial pressure; OSLER, the Oxford sleep resistance test; REM, rapid eye movement; SBP, systolic blood pressure; SWS, slow wave sleep; TST, total sleep time. 
Table 3 Whole and within initial treatment group changes from baseline to week 24

\begin{tabular}{|c|c|c|c|c|c|c|}
\hline & $\begin{array}{l}\text { Overall mean or } \\
\text { median change } \\
(95 \% \mathrm{CI} \text { or IQR) }(n=48)\end{array}$ & p Value & $\begin{array}{l}\text { CPAP mean or median } \\
\text { change }(95 \% \mathrm{Cl} \text { or IQR) } \\
(\mathrm{n}=26)\end{array}$ & p Value & $\begin{array}{l}\text { Sham mean or median } \\
\text { change }(95 \% \mathrm{Cl} \text { or IQR) } \\
(\mathrm{n}=20)\end{array}$ & p Value \\
\hline \multicolumn{7}{|l|}{ OSA and sleepiness } \\
\hline ODI (events/h) & $-29.7(-38.1$ to -16.0$)$ & $<0.0001^{*}$ & $-28.7(-38.1$ to -15.1$)$ & $<0.0001^{*}$ & $-29.7(-51.7$ to -20.7$)$ & $<0.0001^{*}$ \\
\hline Subjective (ESS, scale 0-24) & $-3.7(-4.8$ to -2.6$)$ & $<0.0001$ & $-3.4(-4.7$ to -2.1$)$ & $<0.0001$ & $-4.1(-6.0$ to -2.1$)$ & 0.0004 \\
\hline Objective (modified MWT, min) & $-0.5(-1.3$ to 0.3$)$ & 0.21 & $0.6(-1.7$ to 0.5$)$ & 0.30 & $-0.2(-1.5$ to 1.1$)$ & 0.68 \\
\hline Subcutaneous abdominal fat $\left(\mathrm{cm}^{3}\right)$ & $1.6(-16.4$ to 19.7$)$ & 0.85 & $-0.5(-29.1$ to 28.1$)$ & 0.97 & $4.1(-19.4$ to 27.7$)$ & 0.72 \\
\hline Liver fat (HU) & $-0.2(-2.4$ to 2.0$)$ & 0.83 & $-0.5(-2.0$ to 1.0$)$ & 0.49 & $0.3(-3.1$ to 3.8$)$ & 0.85 \\
\hline Liver/spleen ratio (HU) & $-0.02(-0.07$ to 0.03$)$ & 0.42 & $-0.04(-0.09$ to 0.008$)$ & 0.09 & $0.006(-0.1$ to 0.1$)$ & 0.91 \\
\hline Total fat $(\mathrm{kg})$ & $-0.1(-1.0$ to 0.8$)$ & 0.83 & $-0.7(-3.7$ to 2.3$)$ & 0.63 & $0.5(-0.2$ to 1.1$)$ & 0.16 \\
\hline Lean muscle $(\mathrm{kg})$ & $1.4(0.8$ to 1.9$)$ & $<0.0001$ & $1.2(0.5$ to 1.9$)$ & 0.0018 & $1.7(0.7$ to 2.6$)$ & 0.002 \\
\hline \multicolumn{7}{|l|}{ Metabolic markers } \\
\hline Fasting insulin $(\mu \mathrm{lU} / \mathrm{ml})$ & $-0.88(-2.65$ to 0.90$)$ & 0.32 & $-2.62(-4.85$ to -0.39$)$ & 0.02 & $1.4(-1.3$ to 4.1$)$ & 0.29 \\
\hline Fasting glucose (mmol/litre) & $0.14(0.002$ to 0.28$)$ & 0.05 & $0.13(-0.07$ to 0.33$)$ & 0.18 & $0.15(-0.06$ to 0.36$)$ & 0.15 \\
\hline Leptin (IU/litre) & $-1.05(-3.03$ to 0.94$)$ & 0.29 & $-1.30(-4.03$ to 1.43$)$ & 0.33 & $-0.71(-3.88$ to 2.46$)$ & 0.64 \\
\hline Adiponectin (IU/litre) & $-0.26(-0.82$ to 0.31$)$ & 0.37 & $-0.06(-0.57$ to 0.45$)$ & 0.81 & $-0.52(-3.88$ to 0.68$)$ & 0.38 \\
\hline
\end{tabular}

Bold values are statistically significant $(\mathrm{p}<0.05)$

Data are whole and within-group mean or median differences from baseline (and $95 \%$ Cls or IQRs) as appropriate. The significance of the differences was assessed by $t$ test after normalisation as required, or by rank-sum test as indicated with *.

AHI, apnea hypopnoea index; CPAP, continuous positive airway pressure; ESS, Epworth Sleepiness Scale; HOMA, homeostasis model assessment of insulin resistance; HU, Hounsfield unit; ISx, insulin sensitivity as measured by minimal model analysis; MWT, modified wakefulness test; ODI, oxygen desaturation index; QUICKI, quantitative insulin sensitivity check index; VAF, visceral abdominal fat.

body weight alone would be expected to reduce VAF and metabolic syndrome. This finding that 3 months of CPAP can reduce total body weight has never previously been reported in any randomised sham-controlled trial and requires replication in other cohorts.

There was also no effect of CPAP on liver fat, which is in contrast to a previous uncontrolled study that showed $2-3$ years of CPAP treatment can significantly decrease liver fat, but only in the subgroup of CPAP adherers $(n=6)$ in whom other lifestyle modifications undertaken in conjunction with CPAP could have plausibly reduced liver fat. ${ }^{15}$ Furthermore the changes observed in this highly selected patient group may not be comparable to a general OSA population.

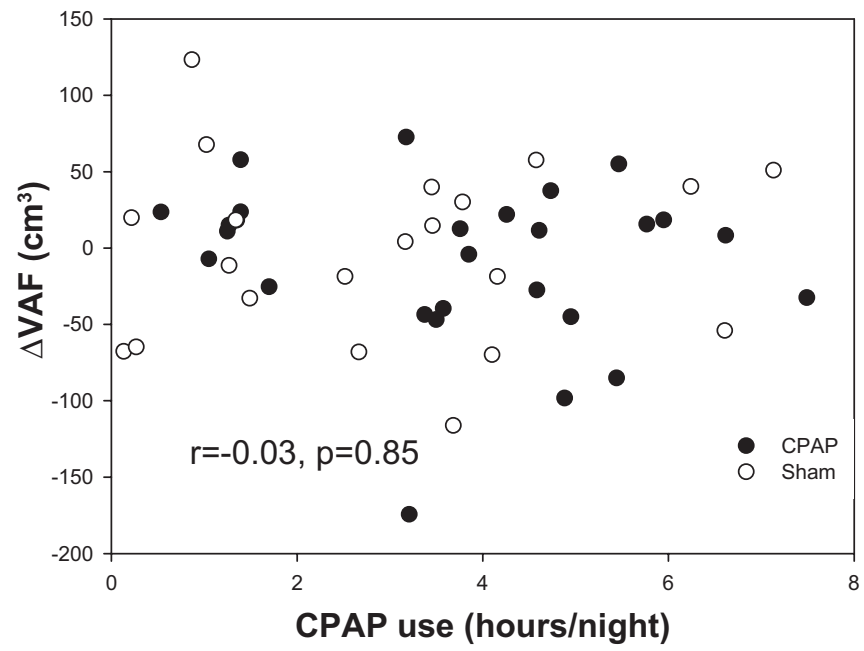

Figure 6 Correlation between continuous positive airway pressure (CPAP) use and the change in visceral abdominal fat (VAF) at week 12. The $r$ and $p$ values are derived from Pearson's correlations.
We found that CPAP therapy did not alter total body fat at any time, but significantly increased lean (muscle) mass at 6 months in all participants irrespective of initial treatment allocation. Our total body findings are consistent with an uncontrolled study showing that 8 months of CPAP did not alter total body fat but did significantly increase lean muscle mass. ${ }^{12}$ Increased lean body mass can have positive metabolic benefits if persistent over time and of sufficient magnitude. Potential improvements include increased exercise capacity, basal metabolic rate and ISx. Such improvements would occur even without changes in total or regional body fat.

The ISx findings are consistent with all previous randomised sham-controlled trials investigating 6-12 weeks of CPAP therapy. ${ }^{16} 173435$ Although one sham-controlled trial showed improvement in ISx after just 1 week, ${ }^{14}$ a recent meta-analysis of randomised controlled studies ${ }^{36}$ (which included that trial) is consistent with our findings of no CPAP effect on ISx. Since the randomised controlled portion was of 1 -week duration, that study was not designed to determine whether the improvement in ISx is maintained longer term. In contrast, we did not assess acute changes in ISx, so it remains possible that CPAP could acutely and transiently improve glucose metabolism.

Small long-term improvements in ISx were observed in those who received a total of 6 months of CPAP treatment, although there were no between-group differences. Other uncontrolled trials investigating the effect of CPAP on glucose metabolism of at least 6 months' duration are available ${ }^{11} 37-40$ and report conflicting changes. Of these, only one formally measured ISx and this also showed an improvement. ${ }^{40}$ Our data, together with the published literature, suggest that CPAP therapy exceeding 3 months is required to improve ISx. We acknowledge that these longer-term analyses are uncontrolled and therefore a randomised controlled study of sufficient duration is needed to prove this. 
Our findings indicate that CPAP treatment does not change adiponectin levels and this finding is consistent with two previous randomised sham-controlled trials. ${ }^{1641}$ We also did not observe a change in leptin after CPAP, but did find a significant correlation between the change in BMI and leptin levels. This suggests that the association between OSA and leptin is mediated through obesity, as previously suggested. ${ }^{42}$ Our results are also consistent with some ${ }^{43}{ }^{44}$ but not other studies. ${ }^{45} 46$ Additionally there was no between-group difference in subjective sleepiness observed, however our participants were only mildly sleepy at baseline. Other randomised sham-controlled trials have also reported similar mild sleepiness at baseline. ${ }^{47} 48$

A strength of this study is the inclusion of a sham-control arm. A control group, blinded to treatment allocation, is important as participants enrolled in a study may alter behaviours, such as diet and exercise, as they are aware they are being monitored. These lifestyle changes could well alter many of the markers of metabolic function which were our primary outcomes. For these reasons, a sham control was considered essential.

The CPAP adherence observed in this study is comparable to other sham-controlled studies. ${ }^{16} 1747$ CPAP adherence has been defined as the use of more than $4 \mathrm{~h}$ per night, five nights per week, which is equivalent to approximately $3 \mathrm{~h}$ per night. Our adherence rate was $3.6 \mathrm{~h}$ per night in the treatment arm. Our study was designed as a practical, intention-to-treat study that in turn could be generalisable to the greater OSA population. We did not include subjects based on their compliance nor did we include a run-in treatment arm to exclude the non-adherers which has been the case in other mechanistic studies. ${ }^{35}$ Our aim was to represent the general OSA population which we believe we have done as our rates are comparable to those of 'real life' CPAP studies that investigate methods to increase adherence. ${ }^{49}$ Additionally our rates $(3.6 \mathrm{~h} / \mathrm{night})$ are comparable to those reported by another 3-month sham-controlled study (3.3 h/ night) investigating ISx. ${ }^{16}$ Interestingly, CPAP use increased by $1.1 \mathrm{~h}$ in those who initially received sham treatment, but was not significantly increased in those who initially received CPAP therapy. This illustrates the importance of recruiting CPAP-naive individuals into a parallel rather than crossover study, as we have done here. A sham control is critical since placebo effects on subjective measures such as sleepiness were detected and have also previously been reported. ${ }^{48}$ Not only is our CPAP compliance comparable with other studies, but we also showed that CPAP compliance per se (as well as age, BMI, waist circumference and sleepiness) did not influence the treatment effect. Furthermore, the change in VAF that can be expected is small and demonstrating such a change would require a study of 5000 men assuming $80 \%$ power and two-sided $\alpha$ of 0.05 (post hoc analysis). Such a sham-controlled randomised controlled trial would be difficult to perform.

In conclusion, this study demonstrated that CPAP alone does not reduce visceral adiposity, even in the longer term. Obese men with severe OSA will most likely require weightloss interventions in conjunction with CPAP treatment to achieve this. Although 3 months of CPAP treatment did not improve ISx, longer-term treatment may produce beneficial changes but this requires further investigation.

Acknowledgements We would like to thank Jonathan Poh, George Dungan, Frances Clements, Kerri Melehan and the sleep physicians and technicians at the Woolcock Institute of Medical Research and Royal Prince Alfred Hospital, Sleep Disorders Unit. We would also like to thank the men who participated in the study. We thank Bill Hardy, Phillips-Respironics, for the supply of manufactured sham devices.
Contributors All authors equally contributed to the concept and design of the trial PYL and RRG obtained funding. CMH was responsible for data acquisition, statistical analysis and the preparation of the manuscript. All authors participated in the overall analysis and interpretation of the data, revision of the manuscript and provided final approval of the submitted version.

Funding Supported by the National Health and Medical Research Council of Australia (NHMRC) through a project grant (512498), a Centre for Clinical Research Excellence in Interdisciplinary Sleep Health (571421) and fellowships to CH, RK, CP, RRG and PYL (512057, 633161, 571179, 202916 and 511929, respectively). Sham machines were provided by Phillips Respironics.

\section{Competing interests None.}

Ethics approval Ethics approval was provided by Sydney South West Area Health Service Human Research and Ethics Committee (RPAH Zone).

Provenance and peer review Not commissioned; externally peer reviewed.

\section{REFERENCES}

1. Young T, Peppard PE, Taheri S. Excess weight and sleep-disordered breathing. J App/ Physiol 2005;99:1592-9.

2. Marshall NS, Wong KK, Liu PY, et al. Sleep apnea as an independent risk factor for all-cause mortality: the Busselton Health Study. Sleep 2008;31:1079-85.

3. Young T, Finn L, Peppard PE, et al. Sleep disordered breathing and mortality: eighteen-year follow-up of the Wisconsin sleep cohort. Sleep 2008;31:1071-8.

4. Punjabi NM, Caffo BS, Goodwin JL, et al. Sleep-disordered breathing and mortality: a prospective cohort study. PLoS Med 2009;6:e1000132.

5. Levy $\mathbf{P}$, Bonsignore MR, Eckel J. Sleep, sleep-disordered breathing and metabolic consequences. Eur Respir J 2009;34:243-60.

6. Kuk JL, Katzmarzyk PT, Nichaman MZ, et al. Visceral fat is an independent predictor of all-cause mortality in men. Obesity (Silver Spring) 2006;14:336-41.

7. Yusuf S, Hawken S, Ounpuu S, et al. Obesity and the risk of myocardial infarction in 27,000 participants from 52 countries: a case-control study. Lancet 2005;366:1640-9.

8. Bonora $\mathbf{E}$, Kiechl S, Willeit J, et al. Insulin resistance as estimated by homeostasis model assessment predicts incident symptomatic cardiovascular disease in Caucasian subjects from the general population: the Bruneck study. Diabetes Care 2007:30:318-24

9. Marin JM, Carrizo SJ, Vicente E, et al. Long-term cardiovascular outcomes in men with obstructive sleep apnoea-hypopnoea with or without treatment with continuous positive airway pressure: an observational study. Lancet 2005:365:1046-53.

10. Trenell MI, Ward JA, Yee BJ, et al. Influence of constant positive airway pressure therapy on lipid storage, muscle metabolism and insulin action in obese patients with severe obstructive sleep apnoea syndrome. Diabetes Obes Metab 2007;9:679-87.

11. Chin K, Shimizu K, Nakamura T, et al. Changes in intra-abdominal visceral fat and serum leptin levels in patients with obstructive sleep apnea syndrome following nasal continuous positive airway pressure therapy. Circulation 1999;100:706-12.

12. Munzer T, Hegglin A, Stannek T, et al. Effects of long-term continuous positive airway pressure on body composition and IGF1. Eur J Endocrinol 2010;162:695-704

13. Vgontzas AN, Zoumakis E, Bixler EO, et al. Selective effects of CPAP on sleep apnoea-associated manifestations. Eur J Clin Invest 2008;38:585-95.

14. Lam JC, Lam B, Yao TJ, et al. A randomised controlled trial of nasal continuous positive airway pressure on insulin sensitivity in obstructive sleep apnoea. Eur Respir J 2010;35:138-45.

15. Shpirer I, Copel L, Broide E, et al. Continuous positive airway pressure improves sleep apnea associated fatty liver. Lung 2010;188:301-7.

16. West SD, Nicoll DJ, Wallace TM, et al. Effect of CPAP on insulin resistance and $\mathrm{HbA} 1 \mathrm{c}$ in men with obstructive sleep apnoea and type 2 diabetes. Thorax 2007:62:969-74

17. Coughlin SR, Mawdsley L, Mugarza JA, et al. Cardiovascular and metabolic effects of CPAP in obese males with OSA. Eur Respir J 2007:29:720-7.

18. Bergman RN, Ader M, Huecking $\mathrm{K}$, et al. Accurate assessment of beta-cell function: the hyperbolic correction. Diabetes 2002;51(Suppl 1):S212-20.

19. Utzschneider KM, Prigeon RL, Faulenbach MV, et al. Oral disposition index predicts the development of future diabetes above and beyond fasting and 2-h glucose levels. Diabetes Care 2009;32:335-41.

20. Phillips CL, Yee BJ, Marshall NS, et al. Continuous positive airway pressure reduces post prandial lipidemia in obstructive sleep apnea. A randomised, placebo controlled crossover trial. Am J Respir Crit Care Med 2011;184:355-61.

21. Kushida CA, Nichols DA, Quan SF, et al. The Apnea Positive Pressure Long-term Efficacy Study (APPLES): rationale, design, methods, and procedures. J Clin Sleep Med 2006;2:288-300.

22. Johns MW. A new method for measuring daytime sleepiness: the Epworth Sleepiness Scale. Sleep 1991;14:540-5.

23. Demerath EW, Ritter KJ, Couch WA et al. Validity of a new automated software program for visceral adipose tissue estimation. Int J Obes (Lond) 2007;31:285-91.

24. Phillips CL, Yee BJ, Trenell Ml, et al. Changes in regional adiposity and cardiometabolic function following a weight loss program with sibutramine in obese men with obstructive sleep apnea. J Clin Sleep Med 2009:5:416-21. 
25. Lohmann TG, Roche AF, Martorell R. Anthropometric Standardization Reference Manual. Champaign, Illinois: Hunam Kinetic Books, 1988.

26. Dalla Man C, Caumo A, Basu R, et al. Minimal model estimation of glucose absorption and insulin sensitivity from oral test: validation with a tracer method. Am J Physiol Endocrinol Metab 2004;287:E637-43

27. Matthews DR, Hosker JP, Rudenski AS, et al. Homeostasis model assessment: insulin resistance and beta-cell function from fasting plasma glucose and insulin concentrations in man. Diabetologia 1985;28:412-19.

28. Katz A, Nambi SS, Mather K, et al. Quantitative insulin sensitivity check index: a simple, accurate method for assessing insulin sensitivity in humans. J Clin Endocrinol Metab 2000;85:2402-10.

29. Diabetes Australia. Diabetes Management in General Practice: Guidelines for Type 2 Diabetes. 17th edn. 2011-2012. http://www.diabetesaustralia.com.au/PageFiles/ 763/Diabetes\%20Management\%20in\%20General\%20Practice\%202011-12.pdf (accessed 24 Apr 2012).

30. Foster GD, Borradaile KE, Sanders MH, et al. A randomized study on the effect of weight loss on obstructive sleep apnea among obese patients with type 2 diabetes: the Sleep AHEAD study. Arch Intern Med 2009;169:1619-26.

31. McMillan DE. A very low calorie diet plus lifestyle counselling improved mild obstructive sleep apnoea in overweight patients. Evid Based Nurs 2009;12:111.

32. Tuomilehto HP, Seppa JM, Partinen MM, et al. Lifestyle intervention with weight reduction: first-line treatment in mild obstructive sleep apnea. Am J Respir Crit Care Med 2009:179:320-7.

33. James WP, Caterson ID, Coutinho W, et al. Effect of sibutramine on cardiovascular outcomes in overweight and obese subjects. N Engl J Med 2010;363:905-17.

34. Sharma SK, Agrawal S, Damodaran D, et al. CPAP for the metabolic syndrome in patients with obstructive sleep apnea. N Engl J Med 2011;365:2277-86.

35. Weinstock TG, Wang X, Rueschman M, et al. A controlled trial of CPAP therapy on metabolic control in individuals with impaired glucose tolerance and sleep apnea. Sleep. In press. http://www.journalsleep.org/AcceptedPapers/SP-505-11.pdf

36. Hecht L, Mohler R, Meyer G. Effects of CPAP-respiration on markers of glucose metabolism in patients with obstructive sleep apnoea syndrome: a systematic review and meta-analysis. Ger Med Sci 2011;9:Doc20.

37. Steiropoulos $\mathbf{P}$, Papanas N, Nena $\mathbf{E}$, et al. Markers of glycemic control and insulin resistance in non-diabetic patients with obstructive sleep apnea hypopnea syndrome: does adherence to CPAP treatment improve glycemic control? Sleep Med 2009:10:887-91.
38. Ip MS, Lam KS, Ho C, et al. Serum leptin and vascular risk factors in obstructive sleep apnea. Chest 2000;118:580-6.

39. Lindberg $\mathbf{E}$, Berne C, Elmasry A, et al. CPAP treatment of a population-based sample-what are the benefits and the treatment compliance? Sleep Med 2006; 7:553-60.

40. Schahin SP, Nechanitzky T, Dittel C, et al. Long-term improvement of insulin sensitivity during CPAP therapy in the obstructive sleep apnoea syndrome. Med Sci Monit 2008;14:CR117-21.

41. Kohler M, Ayers L, Pepperell JC, et al. Effects of continuous positive airway pressure on systemic inflammation in patients with moderate to severe obstructive sleep apnoea: a randomised controlled trial. Thorax 2009:64:67-73.

42. Sharma SK, Kumpawat S, Goel A, et al. Obesity, and not obstructive sleep apnea, is responsible for metabolic abnormalities in a cohort with sleep-disordered breathing. Sleep Med 2007:8:12-17.

43. Murri M, Alcazar-Ramirez J, Garrido-Sanchez L, et al. Oxidative stress and metabolic changes after continuous positive airway pressure treatment according to previous metabolic disorders in sleep apnea-hypopnea syndrome patients. Trans/ Res 2009;154:111-21.

44. Drummond M, Winck JC, Guimaraes JT, et al. Autoadjusting-CPAP effect on serum leptin concentrations in obstructive sleep apnoea patients. BMC Pulm Med 2008;8:21.

45. Cuhadaroglu C, Utkusavas A, Ozturk L, et al. Effects of nasal CPAP treatment on insulin resistance, lipid profile, and plasma leptin in sleep apnea. Lung 2009;187:75-81.

46. Harsch IA, Konturek PC, Koebnick C, et al. Leptin and ghrelin levels in patients with obstructive sleep apnoea: effect of CPAP treatment. Eur Respir $J$ 2003:22:251-7.

47. Duran-Cantolla J, Aizpuru F, Montserrat JM, et al. Continuous positive airway pressure as treatment for systemic hypertension in people with obstructive sleep apnoea: randomised controlled trial. BMJ 2010;341:c5991.

48. Hui DS, To KW, Ko FW, et al. Nasal CPAP reduces systemic blood pressure in patients with obstructive sleep apnoea and mild sleepiness. Thorax 2006:61:1083-90.

49. Sparrow D, Aloia M, Demolles DA, et al. A telemedicine intervention to improve adherence to continuous positive airway pressure: a randomised controlled trial. Thorax 2010;65:1061-6. 


\section{Correction}

Hoyos CM, Killick R, Yee BJ, et al. Cardiometabolic changes after continuous positive airway pressure for obstructive sleep apnoea: a randomised sham-controlled study. Thorax 2012;67:1081-1089. doi:10.1136/thoraxjnl-2011-201420

We have since realised that there were two transcription errors included in the manuscript that should ideally be corrected. Since these were transcription errors, neither had any influence on the statistical analyses presented.

- The study flow (figure 1) states that 28 in the CPAP groups and 24 in the sham group completed the 12 week treatment period. Actually, 29 completed CPAP and 23 completed sham CPAP. Furthermore, 2 men in the CPAP group and 3 men in the sham CPAP group withdrew prior to receiving the open-label CPAP treatment after week 12 . The completion numbers of 26 in the CPAP group and 20 in the sham group at week 24 are correct.

- In table 1 and 2 for the results of insulin sensitivity (ISx) a negative sign (ie, '-') was inadvertently left out (all the result should be $10^{-4}$ not $10^{4}$ ). Again this was a transcription error that had no influence on the statistical analyses presented.

Thorax 2013;68:879. doi:10.1136/thoraxjnl-2011-201420corr1 